\title{
VERSÃO BRASILEIRA DA TUCKMAN PROCRASTINATION SCALE: ADAPTAÇÃO E EVIDÊNCIAS PSICOMÉTRICAS बढ
}

\author{
Ricardo Neves Couto ${ }^{a}{ }^{\circledR}$, Patrícia Nunes da Fonseca ${ }^{\text {b1}}{ }^{\circledR}$, Paulo Gregório

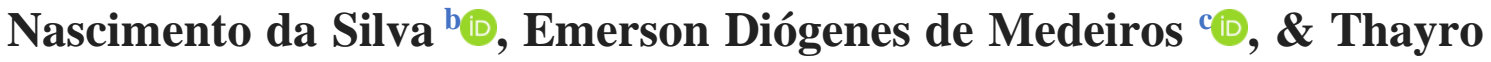 \\ Andrade Carvalho ${ }^{d}{ }^{2}$ 2 \& 3
}

\author{
Universidade Federal do Piauí, Parnaíba, Brasil a ; Universidade Federal

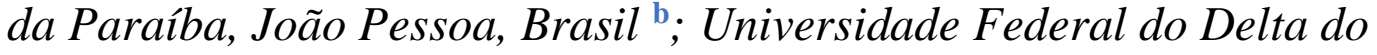 \\ Parnaíba, Parnaíba, Brasil c; Universidade Federal do Sul da Bahia, \\ Teixeira de Freitas, Brasil ${ }^{\mathrm{d}}$.
}

\section{RESUMO}

Objetivou-se reunir evidências de validade e precisão da Tuckman Procrastination Scale (TPS) em contexto brasileiro. Para tanto, foram realizados dois estudos. No Estudo 1, participaram 210 universitários, com idade média de 23,21 ( $D P=7,08)$. Responderam a TPS e questões demográficas. A análise fatorial exploratória sugeriu uma solução unidimensional, com alfa de Cronbach $(\alpha)$ e ômega de McDonald $(\omega)$ iguais a 0,88. No Estudo 2, participaram 274 universitários, com idade média de 23,72 $(D P=7,61)$. Uma análise fatorial confirmatória apontou indicadores adequados (CFI =0,97; TLI =0,96; RMSEA=0,06; Pclose $=0,28)$. Ademais, a confiabilidade foi satisfatória $(\alpha=0,86 ; \omega=0,70)$. Foram encontradas evidências psicométricas para a realidade brasileira, podendo a TPS ser uma ferramenta útil para interessados na temática.

\section{Palabras Claves}

Procrastinação acadêmica; Universitários; Teste psicológico; Validade; Precisão

\section{Abstract}

The objective was to gather evidence of validity and accuracy of the Tuckman Procrastination Scale (TPS) in Brazilian context. Therefore, two studies were carried out. In Study 1, the participants were 210 undergraduate students, with an average age of 23.21 (SD =7.08). They responded to TPS and demographic issues. The exploratory factor analysis suggested a one-dimensional solution, with Cronbach's alpha $(\alpha)$ and McDonald's omega $(\omega)$ equal to .88. In Study 2, the participants were 274 undergraduate students, with an average age of 23.72 ( $\mathrm{SD}=7.61)$. A factor confirmatory analysis indicated adequate indicators $(\mathrm{CFI}=$ $.97, \mathrm{TLI}=.96, \mathrm{RMSEA}=.06$, Pclose $=.28)$. In addition, the reliability was satisfactory $(\alpha=.86, \omega=.70)$. Psychometric evidences were found for the Brazilian reality, and TPS could be a useful tool for those interested in the subject.

\section{Keywords}

Academic procrastination; Undergraduate students; Psychological test; Shelf life; Accuracy

\footnotetext{
1 Correspondence about this article should be addressed to Ricardo Neves Couto: r.nevescouto@gmail.com

2 Financial support: This article was supported by CNPq and CAPES through funding of research projects of the second and fourth authors, in addition to a PhD scholarship (CAPES) to the first. We take this opportunity to show our gratitude to these institutions.

3 Conflicts of Interest: The authors declare that the research was conducted in the absence of any commercial or financial relationships that could be construed as a potential conflict of interest.
} 


\section{BRAZILIAN VERSION OF THE TUCKMAN PROCRASTINATION SCALE: ADAPTATION AND PSYCHOMETRIC EVIDENCE}

\section{Introdução}

A procrastinação é um fenômeno que afeta as atividades das pessoas cotidianamente, presente no dia a dia quando deixam de fazer atividades produtivas para passar o tempo de maneira ociosa. Refere-se a decisão de adiar tarefas ou compromissos (Blouin-Hudon \& Pychyl, 2015), o que pode ocasionar prejuízos ao indivíduo visto que, na sociedade ocidental, as diferentes atividades devem ser planejadas e realizadas em um determinado prazo satisfatório, para que haja consequências positivas (Días-Morales, 2019). O ato de procrastinar também pode acarretar sofrimento psicológico a pessoa, pois exige o cumprimento de uma atividade próximo do final do prazo, gerando ansiedade e estresse, ou o não cumprimento e o consequente sofrimento pela dor do fracasso ou/e da crítica de terceiros (Beswick et al., 1988). Por vezes, realizam as tarefas, todavia acabam optando pelas consideradas fáceis, visando alcançar êxito sem muito esforço (Quant \& Sánchez, 2012).

Geralmente, as pessoas que procrastinam para atingir ganhos imediatos, tendem a experimentar maior satisfação momentânea, iniciando o comportamento de procrastinação quando precisam cumprir uma atividade avaliada como difícil, que gera níveis elevados de raiva e ansiedade (Tckman, 1990). Em seguida, optam por condutas de evitação destas tarefas, a de fim obter um alívio, ao postergar a sua execução (Furlán et al., 2015). Segundo Quant e Sánchez (2012), existem dois tipos de procrastinadores: (1) os ativos, que ignoram completamente uma determinada tarefa, optando por não concretizá-la e (2) os passivos, que tendem a realizar a atividade, mesmo que de maneira imprecisa e deliberada. De acordo com Chun e Choi (2005) esses dois grupos apresentam comportamentos similares, diferindo apenas na forma de administrar o tempo, além da percepção de autoeficácia referentes aos estilos de enfrentamentos na realização de uma atividade. Ademais, existem distintas classificações acerca da procrastinação (Yazıcı \& Bulut, 2015). Por exemplo, Klingsieck (2013) aponta quatro perspectivas evidentes: (1) Psicologia diferencial, que compreende a procrastinação como um traço de personalidade; (2) Psicologia motivacional e de volição, que entende como um fracasso na motivação e/ou nos aspectos volitivos (querer/ vontade); (3) Psicologia clínica, dedicando as variáveis que são clinicamente relevantes para a procrastinação, tais como, transtornos de personalidade, ao estresse, ansiedade, ansiedade e depressão; e, por fim, 
(4) situacional, que investiga os aspectos contextuais, e tem se tornado cada vez mais popular.

A presente pesquisa foca-se na procrastinação situacional, que pode ser entendida como uma tendência a adiamentos frequentes de aspectos específicos da vida de uma pessoa (Yockey, 2016), afetando o desempenho acadêmico, social e psicológico, além do seu bem-estar (Kim \& Seo, 2015), sendo um fenômeno, particularmente, comum entre estudantes universitários (Balkis \& Duru, 2019). Tendo em conta aquilo que foi anteriormente citado, pode-se assumir que a procrastinação, especificamente a acadêmica, foco desta pesquisa, é um hábito destrutivo no cotidiano das pessoas com consequências que extrapolam o contex to de estudo (Blouin-Hudon \&. Pychyl, 2015).

A procrastinação acadêmica (PA) é frequentemente definida como uma característica comportamental ou disposição para adiar ou atrasar de forma voluntária a tomada de decisões e/ou a execução de tarefas acadêmicas ou curso pretendido relacionado ao estudo (Steel \& Klingsieck 2016), isto é, um atraso intencional e desnecessário frente ao início ou conclusão de atividades relacionadas ao estudo. Este fenômeno minimiza a chance de os objetivos serem atingidos e pode causar diferentes problemas em aspectos da vida (e.g. saúde, financeira, relações sociais; Mortazavi et al., 2015). Tendo em conta o âmbito acadêmico, Vargas (2017) demonstra em sua revisão, que cerca de 70 e $95 \%$ dos estudantes universitários procrastinam ocasionalmente e entre 20 a 40\% o fazem de forma crônica. Em uma investigação com 169 universitários peruanos que cursavam educação em línguas, literatura e comunicação, Pérez e Acuña (2016) apontaram um grau de procrastinação de $71 \%$ na amostra considerada, destes $63,9 \%$ alcançaram elevados níveis e 15,4\% pontuaram baixos. Além disso, os autores observaram que quanto maior o grau de escolarização, ou avanços no curso, menor será o nível de PA, tendo em vista que as cobranças ficam maiores e os prazos ficam mais curtos.

Essa manifestação específica de procrastinação é entendida como um comportamento de evitação de trabalhos acadêmicos, em que o estressor é a obrigação da realização de uma tarefa e o indivíduo opta por adiá-la (Steel \& Klingsieck 2016). Tal decisão é atribuída a uma resposta emocional negativa frente à sua execução; caracterizando-se como uma reação temporária que pode ser causada por uma combinação de descrença da pessoa em realizar bem tarefas e a frequente atribuição da culpa para fontes externas (Tuckman, 1990). Ademais, é possível inferir três manifestações comportamentais específicas da procrastinação: (1) falta de rapidez na 
intenção ou no comportamento; (2) discrepância entre intenção/comportamento; e (3) a preferência por atividades concorrentes e mais prazerosas (Schouwenburg, 1995). Evidencia-se que tal conduta pode afetar a médio e longo prazo a vida das pessoas (Clariana et al., 2011), e estão relacionadas dificuldades acadêmicas, como estratégias de aprendizagem menos eficazes (Goroshit, \& Hen, 2019) ou má regulação do comportamento (Eisenbeck et al., 2019). Consequentemente a PA pode estar relacionada com a ansiedade frente a uma avaliação (Furlán et al., 2015; Y1ldırım \& Demir, 2019), ao baixo desempenho acadêmico (Semprebon et al., 2017), percepções inadequadas quanto estudante (Silva et al., 2020), o que acaba interferindo no bem estar físico e mental (Duru \& Balkis, 2017).

Quanto a tarefa de mensuração da PA, verifica-se na literatura algumas medidas (ver Díaz-Morales, 2019). Por exemplo, em seu estudo de revisão Quant e Sánchez (2012) indicaram três instrumentos que têm apresentado indícios de validade e confiabilidade aceitáveis: (1) Aitken Procrastination Inventory, (API; Aitken ,1982), composta inicialmente por 19 itens, respondidos em uma escala de 5 pontos, variando de 1 (falso) a 5 (verdadeiro) com um alfa de Cronbach igual a 0,82, avalia a tendência global dos estudantes em postergar tarefas acadêmicas; (2) Procrastination Assessment Scale Students, PASS (Solomon \& Rothblum, 1984), avalia os antecedentes cognitivos e as condutas do ato de procrastinar, tais como o perfeccionismo, a ansiedade, a baixa confiança e a preguiça. É composto por 44 itens, que são respondidos por uma escala de 5 pontos, variando de 1 (nunca) a 5 (sempre). Sua confiabilidade foi testada por Ferrari (1989), por meio da técnica do teste-reteste, a qual teve uma forte correlação $(r=0,81, p$ $<$ 0,01) e (3) Tuckman Procrastination Scale (TPS; Tuckman, 1991), objeto de interesse do presente estudo, foi escolhido porque é parcimonioso, com bons indicadores psicométricos e por ter uma constituição teórica que corrobora com os interesses da presente investigação. Portanto, a seguir, descreve-se brevemente acerca dessas considerações.

Tuckman (1990) considera a PA um fenômeno referente à ausência de autocontrole, ou seja, uma tendência de se evitar ou ignorar as tarefas que deveriam realizar. Para este autor, a procrastinação é causada por uma combinação da descrença do aluno na realização de tarefas, a necessidade de gratificação momentânea e a frequente atribuição de culpa a fontes externas em "situações difíceis". A partir desse pressuposto, Tuckman, (1991) desenvolveu a Tuckman Procrastination Scale (TPS). Em seu estudo contou com 233 estudantes universitários estadunidenses e apresentou a versão inicial 
composta por 72 itens, que avaliavam três características (tendências), a saber: (a) o adiamento do início de tarefas, (b) a evitação em fazer coisas que são consideradas pessoalmente desagradáveis e (c) a culpa que o indivíduo atribui aos demais, por seus infortúnios. Por meio de uma análise dos componentes principais a medida foi reduzida para 35 itens e precisão (alfa de Cronbach; $\alpha$ ) de 0,86 . Com base nesse estudo, foi sugerido uma versão mais parcimoniosa, composta por 16 itens, com uma estrutura unidimensional, que explicou $30 \%$ da variância total da medida, com precisão avaliada pelo alfa de Cronbach, de 0,90.

Além disso, pode-se observar que TPS é usada para avaliar a tendência geral em perder tempo e protelar a realização das tarefas em estudos de diferentes países, sendo corroborado a estrutura unifatorial do instrumento. Por exemplo, visando de obter novos parâmetros psicométricos da TPS, Furlán et al. (2012) consideraram uma amostra de 210 estudantes universitários e adaptaram a TPS para o contexto argentino. Nesse estudo consideraram 15 itens. Assim, após os autores realizarem validação semântica, análises fatoriais (exploratória e confirmatória), ao final, verificou-se índices de ajustes satisfatórios $(\mathrm{GFI}=0,98 ; \mathrm{CFI}=0,99$ e RMSEA $=0,05)$, corroborando a estrutura unidimensional e adequada consistência interna $(\alpha=0,94)$. Além disso, averiguou-se a validade de critério por meio do rendimento acadêmico, com o qual apresentou correlação negativa $(r=-0,22, p<0,01)$.

De maneira similar, em pesquisa realizada por Özer, et al. (2009) com 858 universitários turcos, consideraram dois estudos: no primeiro realizou-se uma análise fatorial exploratória categórica, que indicou uma estrutura unidimensional, $\operatorname{com} \alpha=0,90$, que foi corroborada no segundo estudo, por meio do método de estimação mínimos quadrados robustos ponderados $(\mathrm{NNFI}=0,94 ; \mathrm{CFI}=0,94 ; \mathrm{RMSEA}=0,07)$ e precisão $(\alpha)$ de 0,85 . Além disso a mediada se correlacionou negativamente com autoeficácia acadêmica e autoestima. Já em estudo realizado na Malásia por Baka e Khan (2016), verificou que autoeficácia acadêmica foi influenciada negativamente pela PA, e a TPS indicou uma precisão $(\alpha)$ de 0,76 . Por fim, em contexto peruano, contando com uma amostra composta por 764 universitários, Alegre-Bravo e Benavente-Dongo (2020) corroboraram a estrutura interna da TPS, propondo uma versão reduzida do instrumento (reunindo 12 itens), que se relacionou negativamente com desempenho acadêmico.

Observa-se, pois, que apesar de diferentes versões da TPS terem sido utilizadas em estudos que consideram a procrastinação, sua prevalência e correlatos em amostras e países distintos, não foram encontrados estudos em português brasileiro, que utilizassem 
tal instrumento, o que demonstra uma lacuna no cenário nacional, fato que justifica os esforços angariados para a execução desta pesquisa. Assim, dado o exposto até o momento, julga-se importante contar com instrumentos válidos, que colaborem para a sistematização e entendimento da procrastinação. Logo, este estudo tem como objetivo adaptar a Tuckman Procrastination Scale para o português brasileiro, reunindo evidências de validade e precisão. Para tanto, levou-se a cabo dois estudos empíricos, a serem apresentados a seguir.

\section{Método}

\section{Participantes}

Contou-se com uma amostra total, não probabilística (de conveniência) de 484 universitários ( $M_{\text {idade }}=23,50$; amplitude 18 a 62 anos), dos estados de Pernambuco $(43,4 \%)$ e Paraíba $(56,6 \%)$. Estes eram em maioria do sexo feminino $(64,5 \%)$, oriundos de instituições públicas $(76,6 \%)$ e cursando Psicologia (54,8\%). A seguir, serão apresentados os perfis sociodemográficos dos participantes, que foram divididos em duas amostras distintas, que compuseram o estudo 1 (N1) e o estudo (N2).

$\mathrm{N}_{1}$ - Contou-se com uma amostra não-probabilística (por conveniência) de 210 estudantes de instituição pública de ensino superior da cidade de Petrolina, Pernambuco. Estes tinham em média 23,21 anos de idade ( $D P=6,10$; amplitude 18 a 53 anos), sendo a maioria do sexo feminino $(61,9 \%)$ e solteiros $(89,5 \%)$, cursando Psicologia $(62,5 \%)$.

$\mathrm{N}_{2}$ - Participaram do estudo 274 estudantes, selecionados de maneira nãoprobabilística (por conveniência), de instituições públicas $(59,2 \%)$ e particulares de ensino superior (40,8\%) da cidade de João Pessoa, Paraíba. Os universitários tinham em média 23,72 anos de idade ( $D P=7,61$; amplitude 18 a 62 anos), sendo a maioria do sexo feminino $(67,2 \%)$ e solteiro $(85,7 \%)$, cursando Psicologia $(34,3 \%)$, Farmácia $(10,6 \%)$ e Administração (7,4\%).

\section{Instrumentos}

Os participantes responderam um livreto contento os seguintes instrumentos:

Tuckman Procrastination Scale - (TPS): elaborada por Tuckman (1991), foi adaptada para o contexto Argentino por Furlán et al. (2012). A adaptação argentina da escala ficou composta por 15 itens e é a versão considerada nesta oportunidade, tendo em vista os procedimentos rigorosos e as análises estatísticas robustas na obtenção de seus 
índices psicométricos. Os itens (e.g. "item 08: Sinto que não posso resolver as atividades, mesmo gastando muito tempo tentando. ”) são respondidos em escala de cinco pontos tipo Likert, variando de 1 "Nunca" a 5 "Sempre". Ademais, 4 itens (05, 07, 11 e 14) apresentam pontuação inversa (e.g. "item 14: Sempre término as atividades importantes com tempo de sobra").

Questionário sociodemográfico. Os participantes responderam a um conjunto de perguntas, a exemplo de sexo, idade, estado civil e renda familiar, que foram utilizadas com o objetivo de caracterizar a amostra.

\section{Procedimento}

Inicialmente, a escala foi traduzida para o português brasileiro, por meio do método do Back Translation (Borsa \& Seize, 2017). Assim, a medida foi traduzida por dois tradutores independentes e em seguida retraduzida para o espanhol, por meio de traduções às cegas, visando verificar a equivalência dos itens das duas versões (português e espanhol). Ressalta-se que os tradutores estiveram atentos as diferenças culturais de cada idioma, fazendo adequações e correções nos itens, quando necessário.

Posteriormente, a medida passou pelo processo de validação semântica. Para tanto, contou-se com uma amostra de 20 estudantes universitários, de ambos os sexos, dos primeiros e dos últimos períodos da instituição. Nesta oportunidade, verificaram possíveis ajustes nos itens dos instrumentos, seguindo os procedimentos estabelecidos por Pasquali (2016). Vencida esta etapa, tratou-se de aplicar o livreto em ambiente de sala de aula, porém os estudantes respondiam de maneira individual, levando aproximadamente 20 minutos, em média, para finalizar a participação na pesquisa.

Previamente, com a autorização dos diretores e coordenadores de cada curso da instituição de ensino selecionada para a pesquisa, um aplicador treinado apresentava o Termos de Consentimento Livre e Esclarecido para que os participantes (alunos devidamente matriculados na instituição participante) pudessem autorizar sua participação na pesquisa e responder aos instrumentos. Foi assegurado a todos o caráter anônimo e confidencial das respostas, além disso, era enfatizado o caráter voluntário da participação na pesquisa e que não traria nenhum prejuízo ou bônus aos participantes, e que poderiam desistir a qualquer momento sem ônus. 


\section{Análise de dados}

Por meio do software Factor 10.10.03 (Ferrando \& Lorenzo-Seva, 2017) averiguou-se a dimensionalidade do TPS, tendo em conta os métodos Minimum Average Partial (MAP) e Hull Comparative Fit Index (CFI; Lorenzo-Seva et al., 2011), por meio de uma análise fatorial exploratória categórica ULS (Unweighted Least Squares) e matriz de correlações policóricas. Ressalta-se que o MAP é um método estatístico confiável para retenção de fatores e o método Hull configura-se como um dos melhores na estimação da dimensionalidade de um dado conjunto de itens (Lorenzo-Seva et al., 2011). Além disso, verificou-se a consistência interna (precisão) pelo coeficiente alfa de Cronbach ( $\alpha$ ) com base nas correlações policóricas e pelo ômega $(\omega)$ de McDonald. Para tanto, considerouse a escala de resposta do tipo Likert como categorias ordenadas (Holgado-Tello et al., 2010; Lara, 2014).

Com o software R efetuaram-se análises descritivas para caracterizar a amostra. Utilizando-se do pacote Lavaan (Rossel, 2012) foi executada uma análise fatorial confirmatória (AFC) categórica (ordinal), com estimador Weighted Least Squares Mean and Variance-Adjusted (WLSMV; Muthén \& Muthén, 2014), o qual é implementado na matriz de correlações policóricas e considera a natureza ordinal dos dados. Utilizamos os seguintes indicadores para avaliar a adequação do modelo (Hair et al., 2019; Tabachnick \& Fidell, 2019):

(1) Comparative Fit Index (CFI) - índice comparativo, frequentemente, valores a partir de 0,90 são referências de um modelo ajustado; (2) Tucker-Lewis Index (TLI) apresenta uma medida de parcimônia entre os índices do modelo proposto e do modelo nulo, varia de zero a um, com valores acima de 0,90 como aceitáveis; (3) Root-MeanSquare Error of Approximation (RMSEA) e seu intervalo de confiança de 90\% (IC90\%) - recomenda-se valores entre 0,05 e 0,08, admitindo-se até 0,10; (4) o Pclose é um indicador mais criterioso, testando a hipótese nula de RMSEA $<0,05$, deste modo, recomenda-se o Pclose $>$ 0,05 como indicativo de modelo ajustado. $\mathrm{O}$ pacote rotina psych (Revelle, 2013), disponível no R, foi adotado para avaliar a confiabilidade, considerando os índices alfa de Cronbach e o ômega $(\omega)$ de McDonald. 


\section{Considerações éticas}

Ressalta-se, que foram obedecidos todos os padrões éticos gerais e as orientações previstas na Resolução 466/12 e 510/16 do Conselho Nacional de Saúde e o projeto foi submetido e aprovado pelo Comitê de Ética de Pesquisa de uma IES pública do estado da Paraíba (CAAE: 65939317.7.0000.5188/ Parecer $\mathrm{n}^{\circ}$ 0118/17). Ademais, os autores declaram não haver qualquer conflito de interesses relativos a presente pesquisa.

\section{Resultados}

\section{Estudo 1. Adaptação e evidências de validade e precisão da Tuckman Procrastination} Scale.

Inicialmente, foi realizada uma análise fatorial exploratória, tendo como finalidade conhecer a estrutura fatorial da matriz de correlações policóricas entre os 15 itens do instrumento. Por meio dos resultados do índice de Kaiser-Meyer-Olkin (KMO) = 0,90 e o Teste de Esfericidade de Bartlett, $\chi^{2}(105)=1.299,6 ; p<0,001$, comprovouse a pertinência de realizar a análise fatorial. Os métodos (MAP e Hull) sugeriram uma solução unidimensional, verificado pelo índice de ajuste Global Fit Index (GFI) = 0,98. O valor próprio (autovalor) foi de 5,77 e explicou 38,44\% da variância total dos itens. As cargas fatoriais dos itens do TPS e a consistência interna da escala são apresentadas na Tabela 1.

Em suma, resultados apresentados na Tabela 1 demonstraram evidências satisfatórias acerca da validade da TPS, apresentando estrutura unifatorial. Ressalta-se que o item 08 ("Sinto que não posso resolver as atividades, mesmo gastando muito tempo tentando") foi descartado por não alcançar carga fatorial mínima $|0,30|$. Assim, a medida reuniu 14 itens, com saturações fatoriais variando de 0,41 (item 12, "Gostaria de encontrar uma maneira fácil de ser ágil") a 0,80 (item 02, "Adio o início das coisas que não gosto de fazer"). Ademais, a consistência interna, medida através do coeficiente alfa de Cronbach ( $\alpha$ ) com base em correlações policóricas de 0,88 e ômega de McDonald de 0,88 , é considerada satisfatória para a versão de 14 itens.

Posteriormente, visando assegurar qualidades psicométricas complementares referente a estrutura encontrada nesse estudo, faz-se necessário considerar técnicas mais robustas, a exemplo da Análise Fatorial Confirmatória (AFC). Assim como no primeiro, considerou-se a natureza categórica (ordinal) da medida para escolha do estimador a ser utilizado. Mais detalhes serão descritos a seguir. 
Tabela 1.

Estrutura fatorial da Tuckman Procrastination Scale.

\begin{tabular}{lcc}
\hline \multicolumn{1}{c}{ Itens } & Carga Fatorial & $h^{2}$ \\
\hline 02. Adio o início das coisas que não gosto de fazer. & $0,80^{*}$ & 0,64 \\
15. Embora eu saiba que é importante iniciar uma atividade, demoro começar. & $0,79^{*}$ & 0,63 \\
03. Quando tenho um prazo, espero até o último minuto para cumpri-lo. & $0,74^{*}$ & 0,55 \\
01. Demoro desnecessariamente para terminar o trabalho, mesmo quando eles são & $0,71^{*}$ & 0,51 \\
importantes. & $0,70^{*}$ & 0,49 \\
04. Se alguém faz uma tarefa no trabalho/escola melhor que eu, então sinto que fracassei & $0,62^{*}$ \\
na tarefa inteira. & $0,58^{*}$ & 0,38 \\
06. Se eu fracasso no trabalho/escola, eu sou um fracasso como pessoa. & $0,57^{*}$ \\
09. Quando tem algo que eu acho muito difícil resolver, penso em adiar. & $0,54^{*}$ \\
14. Se eu não fizer o certo o tempo todo, as pessoas não me respeitarão. & 0,33 \\
10. Proponho-me conseguir alguma coisa e, em seguida, não começo ou não termino. & 0,29 \\
05. Começo a trabalhar imediatamente, mesmo em atividades que eu acho desagradável. & $0,52^{*}$ \\
07. Destino o tempo necessário para atividades, apesar de serem chatas & $0,42^{*}$ \\
11. Sempre que eu faço um plano de ação, eu sigo. & $0,46^{*}$ & 0,27 \\
13. Não busco melhorar, mesmo que me chateei comigo mesmo quando não consigo fazer & $0,41^{*}$ \\
$\quad$ as coisas. & $0,41^{*}$ \\
12. Gostaria de encontrar uma maneira fácil de ser ágil. & 0,17 \\
08. Sinto que não posso resolver as atividades, mesmo gastando muito tempo tentando. & 0,23 \\
\hline Número de itens & 14 \\
Valor próprio & 2,91 \\
Variância explicada (\%) & 21,28 \\
Alfa de Cronbach & 0,88 \\
Ômega de McDonald & 0,88 \\
\hline Nota: * item retido no fator. Carga fatorial considerada satisfatória para compor o fator & $0,30 \mid . h^{2}=$ comunalidade.
\end{tabular}

\section{Estudo 2. Comprovação da estrutura fatorial da Tuckman Procrastination Scale (TPS)}

Considerando a estrutura unidimensional da TPS, que ficou composta por 14 itens no Estudo 1, foi realizada uma análise fatorial confirmatória (AFC), adotando o método de estimação WLSMV. Pode-se verificar, assim, que o modelo unifatorial apresentou índices de ajustes adequados: CFI =0,97, TLI =0,96, RMSEA $($ IC90\% $)=0,06(0,04-$ $0,07)$ e Pclose $=0,28$. Ressalta-se que todos os pesos fatoriais (Lambdas $-\lambda$ ) foram positivos e diferentes de zero $(\lambda \neq 0 ; z>1,96, p<0,001)$, como observado na Figura 1.

Como exposto na Figura 1, todos os pesos fatoriais indicam que os itens representam satisfatoriamente o construto, com cargas fatoriais $(\lambda)$ variando de 0,24 (item 11. Sempre que eu faço um plano de ação, eu sigo.) a 0,75 (item 4. Se alguém faz uma tarefa no trabalho/escola melhor que eu, então sinto que fracassei na tarefa inteira.). A confiabilidade (precisão) da medida foi avaliada pelo alfa de Cronbach (considerando correlações policóricas) e pelo ômega de McDonald, apresentando valores iguais a 0,86 e 0,70 , respectivamente. 


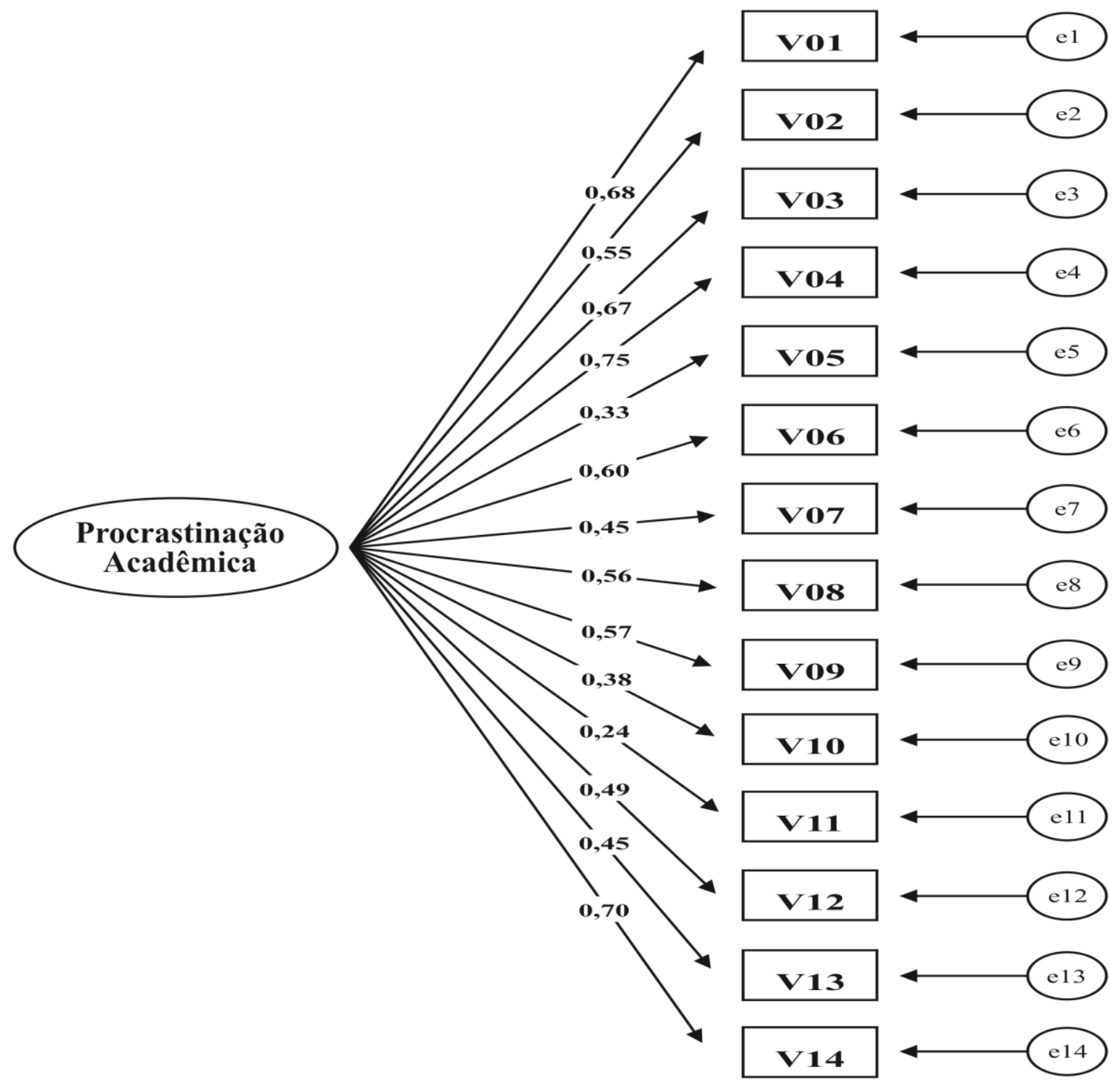

Figura 1. Estrutura unifatorial da Tuckman Procrastination Scale.

\section{Discusión}

A procrastinação, configura-se como um dos fenômenos mais recorrentes no contexto acadêmico, que afeta a esfera social, psicológica e o bem-estar (Kim \& Seo, 2015). Esse fenômeno está relacionado a diversas consequências negativas (e.g., negligências de atividades importantes, perda de oportunidades, etc.), além de está intimamente relacionado com o fracasso acadêmico, uma vez que os estudantes se sentem incapazes de realizar com eficácia as tarefas, adiando-as e, por consequência, afetando o seu desempenho e autoestima (Duru \& Balkis, 2017).

Acredita-se que com os achados e a contemplação do objetivo proposto na presente pesquisa, tem-se uma importante contribuição para a produção da PA, por julgar 
importante contar com instrumentos que apresentem qualidades psicométricas adequadas e fundamentação teórica pertinente, com relevância e aplicabilidade para se compreender seus antecedentes e consequentes. Especificamente, contar com instrumentos com qualidades psicométricas asseguradas no âmbito da PA pode proporcionar estudos com variáveis e contextos que auxiliem na diminuição dos elevados níveis de adiamento ou evitação das atividades acadêmicas (Pérez \& Acuña, 2016).

Tenha-se em conta o supracitado, e compreendendo a necessidade de contar com medidas que possibilitem entender a realidade do procrastinador, o principal objetivo do estudo foi adaptar para o contexto brasileiro uma medida que avalia a procrastinação no âmbito acadêmico (Tuckman, 1991), além de conhecer evidências de validade fatorial e consistência interna da medida. Estima-se que os propósitos dessa pesquisa tenham sido alcançados, pois o instrumento apresentou bons indícios de validade fatorial e de consistência. Entretanto, mesmo que os achados tenham sido promissores, inicialmente, faz-se necessário elencar as limitações potenciais da pesquisa.

Nessa direção, pode-se citar a amostra, uma vez que foi por conveniência (não probabilística), contando com participantes que, convidados, aceitaram colaborar com a pesquisa (Cozby, 2003), fato que não permite que os resultados extrapolem as amostras consideradas, ou seja, de universitários dos estados de Pernambuco e da Paraíba. Dito isto, ressalta-se que não foi objetivo do estudo a generalização dos resultados, mas apresentar uma medida de procrastinação que reunisse evidências que comprovassem a validade e precisão. Considerando essa limitação, sugere-se que estudos futuros abranjam amostras maiores e mais heterogêneas, que considerem outros estados do Nordeste, quiçá outras regiões brasileiras.

Outra limitação potencial refere-se ao fato de ter sido aplicada uma medida de autorrelato (lápis e papel), trazendo algumas desvantagens, pois, uma vez que a procrastinação não é um fenômeno socialmente desejável. Dessa forma, embora a participação na pesquisa fosse anônima, algum grau de desejabilidade social poderia estar envolvido e poderia ter levado a respostas tendenciosas dos respondentes visando a promoção pessoal (Goroshit, \& Hen, 2019).

Em relação aos principais achados desta pesquisa, o Estudo 1, reuniu evidências de validade fatorial e precisão, de acordo com as recomendações da literatura consultada (Hiar et al., 2019; Pasquali, 2016). Assim, para o contexto brasileiro, a TPS apresentouse uma medida reduzida, composta por 14 itens, que foram agrupados em uma única dimensão, denominada de Procrastinação Acadêmica. A consistência interna (precisão), 
foi avaliada por meio do alfa do Cronbach e pelo ômega de McDonald, este último é considerado mais robusto quando comparado ao alfa de Cronbach (Dunn et al., 2014; McDonald, 1999).

No que diz respeito ao Estudo 2, buscou-se reunir evidências psicométricas adicionais. Sendo adotadas análises confirmatórias (AFC; Tabachnick \& Fidell, 2019), considerando a medida categórica (ordinal) estimador Weighted Least Squares Mean and Variance-Adjusted (WLSMV; Muthén \& Muthén, 2014). Para tanto, considerando a estrutura indicada no Estudo 1, foi possível observar adequado ajuste do modelo aos dados (e.g., CFI e TLI > 0,95 e RMSEA < 0,08; Hair et al., 2019; Tabachnick \& Fidell, 2019), além disso, a confiabilidade (precisão), avaliada pelos indicadores de Alfa de Cronbach (matriz policórica) e o ômega de McDonald, apresentaram valores situando entre 0,70 e acima de 0,80, respectivamente, podendo considerá-los meritórios (Taber, 2018). Tais resultados, com estrutura unidimensional, são endossados e corroborados por estudos que tinham o mesmo objetivo deste, considerando amostras de outros países (Alegre-Bravo \& Benavente-Dongo, 2020; Furlán et al., 2012; Özer et al., 2009, Tuckman, 1991).

Acerca de possibilidades futuras, visando superar as limitações aqui encontradas, estudos posteriores poderiam verificar se a estrutura da TPS de 14 itens é confirmada. Nessa direção, seria interessante diversificar as amostras, considerando também diferentes grupos de estudantes, com idades variadas, a exemplo de estudantes de ensino fundamental e médio. Leva-se em conta que estudos tem demonstrado que a idade é uma variável que possivelmente influencie a procrastinação (Beutel et al., 2016).

Ademais, seria relevante reunir evidências de validade convergente-discriminante e de critério, ou ainda levar a cabo estudos que investiguem invariância fatorial, considerando diferentes grupos (gênero, instituição pública/ particular etc.). Uma possível alternativa para minimizar os efeitos da desejabilidade social em estudos de natureza social seria a utilização de medidas implícitas (Gouveia et al., 2012).

Em suma, os resultados, aqui encontrados, indicam evidências da adequação da TPS para a realidade brasileira. É uma medida concisa, composta por 14 itens, com qualidades psicométricas apropriadas, que se propõe a mensurar a tendência para a procrastinação acadêmica em universitários. Pode vir a ser uma ferramenta útil para pesquisadores interessados no tema que desejam conhecer correlatos deste construto, além de poder vir a subsidiar intervenções em instituições de ensino e na clínica. 


\section{Referencias}

Aitken, M. (1982). A personality profile of the college student procrastinator. Unpublished doctoral dissertation, University of Pittsburgh.

Alegre-Bravo, A., \& Benavente-Dondo, D. (2020). Análisis Psicométrico de la Escala adaptada de Procrastinación de Tuckman (APTS). Propósitos y Representaciones, 8(2), e562. http://dx.doi.org/10.20511/pyr2020.v8n2.562

Baka, A. Z, \& Khan U. M. (2016). Relationships Between Self-Efficacy and the Academic Procrastination Behaviour Among University Students in Malaysia: A General Perspectiv. Journal of Education and Learning, 10(3), 265-274. https://doi.org/10.11591/edulearn.v10i3.3990

Beswick, G., Rothblum, E. D., \& Mann, L. (1988). Psychological antecedents of student procrastination. Australian Psychologist, 22(2), 207-217. https://doi.org/10.1080/00050068808255605

Blouin-Hudon, E.-M. C., \& Pychyl, T. A. (2015). Experiencing the temporally extended self: Initial support for the role of affective states, vivid mental imagery, and future self-continuity in the prediction of academic procrastination. Personality and Individual Differences, 86, 50-56. https://doi.org/10.1016/j.paid.2015.06.003

Borsa, J. C., \& Seize, M. M. (2017). Construção e adaptação de instrumentos psicológicos: Dois caminhos possíveis. In J. C. Borsa \& B. F. Damásio, Manual de desenvolvimento de instrumentos psicológicos (pp.15-38). Porto Alegre: Artmed.

Balkis, M., \& Duru, E. (2019). Procrastination and Rational/Irrational Beliefs: A Moderated Mediation Model. Journal of Rational-Emotive \& CognitiveBehavior Therapy, 37, 299-315. https://doi.org/10.1007/s10942-019-00314-6

Beutel, M. E., Klein, E. M., Aufenanger, S., Brähler, E., Dreier, M., ... \& Wölfling, K. (2016) Procrastination, Distress and Life Satisfaction across the Age Range - A German Representative Community Study. PLOS ONE 11(2), e0148054. https://doi.org/10.1371/journal.pone.0148054

Chun, A. H., \& Choi, J. N. (2005). Rethinking procrastination: Positive effects of "active" procrastination behavior on attitudes and performance. The Journal of Social Psychology, 145(3), 245-264. https://doi.org/10.3200/SOCP.145.3.245-264

Clariana, M., Cladellas, R., Badía, M., \& Gotzens, C. (2011). La influencia del género en variables de la personalidad que condicionan el aprendizaje: inteligencia emocional y procrastinación académica. Revista Electrónica Interuniversitaria de Formación del Profesorado, 14(3), 87-96. Retrieved from https://dialnet.unirioja.es/servlet/articulo?codigo $=4625089$

Cozby, P. C. (2003). Métodos de pesquisa em ciências do comportamento. São Paulo: Atlas.

Días-Morales, J. F. (2019). Procrastinación: Una Revisión de su Medida y sus Correlatos. Revista Iberoamericana de Diagnóstico y Evaluación -e Avaliação Psicológica, 51(2), 43-60. https://doi.org/10.21865/RIDEP51.2.04

Dunn, T. J., Baguley, T., \& Brunsden, V. (2014). From alpha to omega: A practical solution to the pervasive problem of internal consistency estimation. British Journal of Psychology, 105(3), 399-412. https://doi.org/10.1111/bjop.12046

Duru, E., \& Balkis, M. (2017). Procrastination, selfesteem, academic performance, and well-being: A moderated mediation model. International Journal of 
Educational Psychology, 6(2), 97-119.

https://doi.org/10.17583/ijep.2017.2584

Ferrando, P. J., \& Lorenzo-Seva, U. (2017). Program FACTOR at 10: Origins, development and future directions. Psicothema, 29(2), 236-240.

https://doi.org/10.7334/psicothema2016.304

Eisenbeck, N., Carreno, D. F., \& Uclés-Juárez, R. (2019). From psychological distress to academic procrastination: Exploring the role of psychological inflexibility. Journal of Contextual Behavioral Science, 13, 103-108. https://doi.org/10.1016/j.jcbs.2019.07.007

Furlán, L., Heredia, D., Piemontesi, S., \& Tuckman, B. (2012). Análisis factorial confirmatorio de la adaptación. Perspectivas em Psicología, 9(3), 142-149. Retrieved from http://www.redalyc.org/articulo.oa?id=483549016020

Furlán, L., Piemontesi, S., Heredia, D., \& Sánchez, R. J. (2015). Ansiedad ante los Exámenes en estudiantes universitarios: Correlatos y Tratamiento. Anuario de Investigaciones de la Facultad de Psicología, 2(1), 37-53. Retrieved from https://revistas.unc.edu.ar/index.php/aifp/article/view/12629

Goroshit, M., Hen, M. (2019). Academic procrastination and academic performance: Do learning disabilities matter?. Current Psychology. https://doi.org/10.1007/s12144-019-00183-3

Gouveia, V. V., Athayde, R. A. A., Mendes, L. A. C., \& Freire, S. E. A. (2012). Introdução às medidas implícitas: Conceitos, técnicas e contribuições. Diaphora - Revista da Sociedade de Psicologia do Rio Grande do Sul, 12, 80-92. Retrieved from http://www.sprgs.org.br/diaphora/ojs/index.php/diaphora/article/view/50

Hair, J. F., Black, W. C., Babin, B. J., \& Anderson, R. E. (2019). Multivariate Data Analysis (8th ed.). Cengage Learning, U.K.

Holgado-Tello, F. P., Chacón-Moscoso, S., Barbero-García, I., \& Vila-Abad, E. (2010). Polychoric versus Pearson correlations in exploratory and confirmatory factor analysis of ordinal variables. Quality \& Quantity, 44(1), 153-166. https://doi.org/10.1007/s11135-008-9190-y

Kim, K. R., \& Seo, E. H. (2015). The relationship between procrastination and academic performance: A meta-analysis. Personality and Individual Differences, 82, 26-33. https://doi.org/10.1016/j.paid.2015.02.038

Klingsieck, K. B. (2013). Procrastination: when good things don't come to those who wait. European Psychologist, 18(1), 24-34. https://doi.org/10.1027/1016$\underline{9040 / \mathrm{a} 000138}$

Lara, S. A. D. (2014). ¿Matrices Policóricas/Tetracóricas o Matrices Pearson? Un estudio metodológico. Revista Argentina de Ciencias del Comportamiento, 6(1), 39-48. Retrieved from http://www.redalyc.org/articulo.oa?id=333430869006

Lorenzo-Seva, U., Timmerman, M. E., \& Kiers, H. A. L. (2011). The Hull Method for Selecting the Number of Common Factors. Multivariate Behavioral Research, 46(2), 340-364. https://doi.org/10.1080/00273171.2011.564527

McDonald, R. P. (1999). Test Theory: A Unified Treatment. Mahwah, NJ: Erlbaum

Mortazavi, F., Mortazavi, S. S., \& Khosrorad, R. (2015) Psychometric Properties of the Procrastination Assessment Scale-Student (PASS) in a Student Sample of Sabzevar University of Medical Sciences. Iranian Red Crescent Medical Journal, 17(9), 28328. https://doi.org/10.5812/ircmj.28328

Muthén, L. K., \& Muthén, B. O. (2014). Mplus user's guide. (Seventh Ed.). Los Angeles: Muthén \& Muthén. 
Özer, B. U., Saçkes, M, \& Tuckman, B. W. (2009). Psychometric Properties of the Tuckman Procrastination Scale in a Turkish Sample. Psychological Reports, 113(3), 874-884. https://doi.org/10.2466/03.20.PR0.113x28z7

Pasquali, L. (2016). TEP - Técnicas de Exame Psicológico: Os Fundamentos. $2^{\mathrm{a}}$ ed. São Paulo, SP: Vetor editora.

Perez, F. T, \& Acuña, J. N. R. (2016). Pérez e Acuña (2016). Procrastinación académica en estudiantes de educación en Lenguas, Literatura y Comunicación: Características, modos y factores. Horizonte de la Ciencia, 6(10), 185-194. Retrieved from http://www.uncp.edu.pe/revistas/index.php/horizontedelaciencia/article/view/ $\underline{214 / 210}$

Revelle, W. (2013) Psych: Procedures for Personality and Psychological Research. Northwestern University, Evanston. Retrieved from http://CRAN.Rproject.org/package $=$ psych

Rossel, Y. (2012). Lavaan: na R package for structural equation modeling. Jounal of Statistical Sofware, 48(2), 1-36. https://doi.org/10.18637/jss.v048.i02

Schouwenburg, H. C. (1995). Academic procrastination: Theoretical notions, measurement, and research. In J. R. Ferrari, J. L. Johnson, \& W. G. McCown (Eds.), Procrastination and task avoidance: Theory, research, and treatment (pp. 71-96). New York, NY: Plenum.

Semprebon, E., Amaro, H. D., \& Beuren, I. M. (2017). A influência da procrastinação no desempenho acadêmico e o papel moderador do senso de poder pessoal. Arquivos Analíticos de Políticas Educativas, 25(20). http://dx.doi.org/10.14507/epaa.25.2545

Silva, P. G. N., Machado, M. O. S, Couto, R. N., Oliveira, L. B. S., \& Fonsêca, P. N. (2020). Motivação para leitura e variáveis sociodemográficas como preditoras da procrastinação acadêmica. Psicología, Conocimiento y Sociedad, 10(1), 139-159. https://dx.doi.org/10.26864/pcs.v10.n1.7

Solomon, L. J., \& Rothblum, E. D. (1984). Academic procrastination: Frequency and cognitive-behavioral correlates. Journal of Counseling Psychology, 31(4), 503-509. https://doi.org/10.1037/0022-0167.31.4.503

Steel, P., \& Klingsieck, K. B. (2016). Academic Procrastination: Psychological Antecedents Revisited. Australian Psychologist, 51(1), 36-46. https://doi.org/10.1111/ap.12173

Quant, D. M., \& Sánchez, A. (2012). Procrastinación, procrastinación académica: concepto e implicaciones. Revista Vanguardia Psicológica Clínica Teórica y Práctica., 3(1), 45-59. Retrieved from https://dialnet.unirioja.es/servlet/articulo?codigo=4815146

Tabachnick, B. G., \& Fidell, L. S. (2019). Using multivariate statistics (7th ed.). Pearson Education.

Taber, K.S. (2018). The Use of Cronbach's Alpha When Developing and Reporting Research Instruments in Science Education. Research in Science Education, 48, 1273-1296. https://doi.org/10.1007/s11165-016-9602-2

Tuckman, B.W. (1990). Group versus goal-setting effects on the self-regulated performance of students differing in selfefficacy. Journal of Experimental Education, 58, 291-298. Retrieved from http://journals.sagepub.com/doi/pdf/10.1177/0013164491512022

Tuckman, B. W. (1991). The Development and Concurrent Validity of the Procrastination Scale. Educational and Psychological Measurement, 51, 473480. https://doi.org/10.1177/0013164491512022 
Vargas, M. A. V. (2017). Academic procrastination: the case of Mexican researchers in psychology. American Journal of Education and Learning, 2(2), 103-120. https://doi.org/10.20448/804.2.2.103.120

Yazıc1, H., \& Bulut, R. (2015). Investigation into the Academic Procrastination of Teacher Candidates' Social Studies with Regard to their Personality Traits. Procedia - Social and Behavioral Sciences, 174, 2270-2277. https://doi.org/10.1016/j.sbspro.2015.01.886

Y1ldırım, F. B., \& Demir, A. (2019). Self-Handicapping Among University Students: The Role of Procrastination, Test Anxiety, Self-Esteem, and SelfCompassion. Psychological Reports, 123(3), 825-843. https://doi.org/10.1177/0033294118825099

Yockey, R. D. (2016). Validation of the Short Form of the Academic Procrastination Scale. Psychological Reports, 118(1), 171-179. https://doi.org/10.1177/0033294115626825 